\title{
A la memoria de Guillermo Soberón, la figura más importante de la salud pública mexicana en el último medio siglo
}

\author{
In memory of Guillermo Soberón, \\ the most important figure in Mexican \\ public health in the last half century
}

\author{
Em memória de Guillermo Soberón, \\ a figura mais importante da saúde pública \\ mexicana na última metade do século
}

Los sistemas de salud son los espacios institucionales que organizan un conjunto de recursos y capacidades para proveer la atención a la salud de la población que la demanda. Para lograr este objetivo, los sistemas de salud requieren que este conjunto de recursos y procesos estén perfectamente coordinados con el fin de generar los productos planteados. Este proceso no es solo técnico sino está cargado de modelos y formas diferentes de organizar un sistema de salud. Generalmente estas decisiones las toman personas con capacidad, entrenamiento y experiencia adecuadas. Las decisiones pueden tener efectos positivos o negativos, intencionados o no intencionados, tanto en el uso adecuado de los recursos como en la salud de las poblaciones atendidas.

Los sistemas de salud no son estáticos, frecuentemente sufren modificaciones y se ajustan a las condiciones cambiantes del entorno. Prácticamente todos los sistemas de salud del mundo se modifican buscando mejorar sus capacidades para poder ampliar la cobertura de servicios, hacer un uso más eficiente de los recursos disponibles, maximizar la calidad de la atención y garantizar los derechos de los usuarios. Para ello se han ejecutado, particularmente en los últimos 40 años, reformas a los sistemas de salud. Aunque las reformas no están libres de valores ideológicos o políticos, en general, buscan promover cambios que fortalezcan el sistema a pesar de que no siempre estén basados sobre la evidencia disponible o que por momentos persigan múltiples motivos además de mejorar las condiciones de salud de la población, como objetivo principal.

La pandemia de COVID-19 que irrumpió en 2020 muestra la fragilidad de los sistemas de salud, particularmente de aquellos en los que la cobertura es segmentada, los mecanismos financieros 
son fragmentados, la calidad de la atención es heterogénea y las estrategias de contención y comunicación comunitaria son inexistentes y/o erráticas. Un elemento más es la capacidad que tienen los tomadores de decisiones de un sistema respecto a sus decisiones estratégicas, particularmente ante la irrupción de un evento como el señalado. La Organización Mundial de la Salud es la agencia global que vigila las condiciones de salud de las poblaciones del mundo y monitorea la emergencia de infecciones que se dispersan rápidamente en la población a fin de sugerir a los países formas de lidiar con este tipo de procesos. Las recomendaciones de la OMS generalmente están basadas en evidencia científica de alta calidad y cuando esta no existe (por los tiempos que requiere generar este tipo de evidencia) utilizan opiniones de expertos reconocidos.

En México, a la fecha la dispersión del COVID-19 ha cobrado la vida de 190,000 personas (según cifras oficiales al 7 de marzo de 2021) y también ha afectado desmedidamente al personal de salud incluido enfermería y otras categorías de personal. De acuerdo a la Organización Panamericana de la Salud, en México y Estados Unidos se contabilizan el 85\% de las muertes por COVID-19 de trabajadores de la salud acaecidas en la región de las Américas. La OPS añade que muchos trabajadores fueron re-dirigidos a la atención del COVID-19 sin la preparación adecuada y sin contar con protección suficiente ${ }^{1}$. Los sistemas de salud deben cuidar a su recurso más importante a través de la capacitación específica, la dotación de equipo de protección y la vinculación laboral de acuerdo a los derechos laborales constitucionales.

Es en este contexto que los aportes de un eminente científico y político precisan enfatizarse. Las reformas del sistema de salud mexicano contaron durante cuatro décadas con la participación de un personaje destacable, que desafortunadamente nos dejó el 12 de octubre de 2020, el Dr. Guillermo Soberón Acevedo. Después de ocupar el cargo de Rector de la Universidad Nacional Autónoma de México por ocho años, el maestro Soberón fue designado coordinador del Plan de Salud de la administración de Miguel De la Madrid (1982-1988). La elaboración del plan implicó un diagnóstico integral del sistema de salud mexicano en momentos en que el país había entrado en la más profunda crisis económica de los últimos 50 años.

Cuando el nuevo gobierno tomó la administración del país, el maestro Soberón nombrado entonces Secretario de Salud. La política de salud definida por Soberón tomó como su primera decisión el cambio a la Ley de Salud vigente para considerar la atención de la salud como un derecho ciudadano y, por lo tanto, como una obligación del estado mexicano de proveerla. La Secretaría de Salud también inició un proceso de descentralización de la estructura de servicios para que las entidades pudieran identificar cercanamente las necesidades de salud de sus poblaciones y administrar los recursos disponibles, tanto federales como los del propio estado.

Una decisión menos conocida, pero también ejecutada desde la lógica soberonista de buscar la integración del sistema de salud en áreas estratégicas, fue la creación de la Comisión Interinstitucional para la Formación de Recursos Humanos en Salud (CIFRHS). Si bien era claro que la integración estructural del sistema no era posible en esos momentos, Soberón buscó integrar funciones sistémicas, en este caso particular, la formación de recursos humanos en salud. El plan lanzado desde la Secretaría de Salud incorporaba no sólo las instituciones del sector, sino también las de educación. Soberón advirtió que integrar ambos sectores era clave para una toma de decisiones prospectiva, que involucrara a los actores interesados con el fin de que los acuerdos fueran asumidos por todos ellos.

Durante su historia, la CIFRHS ha jugado un papel importante para la definición de estándares de entrenamiento, de mecanismos regulatorios de la formación y también ha buscado equilibrar la 
relación entre la producción de profesionales y la capacidad del sistema de salud para incorporarlos laboralmente. Algunos de estos objetivos han sido obtenidos y otros no, pero la CIFRHS representa una institución que ha logrado descentralizarse buscando construir una estructura de decisiones plural.

Una vez que dejó la Secretaría de Salud, Soberón siempre estuvo atento al desarrollo del sistema de salud y de hecho tuvo participaciones importantes. Al final de su período como secretario de salud fue artífice de la creación del Instituto Nacional de Salud Pública (INSP) en 1987, el primer instituto nacional de salud que no tenía como objetivo la atención hospitalaria. Desde su fundación, el INSP ha sido una institución de entrenamiento plural, reconocida internacionalmente, en un campo donde las posiciones epistemológicas e ideológicas son contrastantes. Más tarde, Soberón impulsó la idea de fundar el Instituto Nacional de Medicina Genómica (INMEGEN) y lo hizo de manera práctica buscando recursos financieros y apoyo político dentro del gobierno. El INMEGEN se fundó en 2004 y a la fecha es una institución con logros importantes y con una proyección estratégica para el futuro de la salud pública en el país.

En ese sentido, el recurso más importante en un sistema de salud, es sin duda, el recurso humano. Mientras otros recursos con el tiempo se deprecian, el recurso humano gana valor al incrementar sus capacidades y la experiencia adquirida. La enfermería representa un recurso de gran valor en la producción de servicios a la salud. En la actualidad, las enfermeras se encuentran concentradas en ámbitos hospitalarios en función del modelo de atención prevalente en el sistema de salud mexicano. En un sistema de salud re-orientado hacia la atención primaria a la salud, la enfermería deberá adquirir mayor valor y relevancia a través de todo el sistema.

La enfermería en México ha adquirido un impulso importante en su formación, particularmente de nivel universitario. Anualmente, el país forma alrededor de veinte mil enfermeras egresadas de universidades, con una creciente proporción de egresadas de universidades privadas. Para 2025 tendremos cien mil nuevas enfermeras universitarias, casi una tercera parte de las enfermeras (de todos los niveles de formación) que actualmente trabajan en el sistema de salud del país. Sin embargo, no existe un proceso de planeación de estos egresos con el fin de compatibilizarlo con los requerimientos actuales y futuros del sistema de salud.

Más aún, lo que la epidemia de COVID-19 ha mostrado es que no existen suficientes enfermeras en el país. Si el gobierno tuvo que contratar miles de enfermeras para enfrentar la epidemia es porque la dotación no es suficiente. De acuerdo con datos de la OPS², 87\% de las enfermeras de la región se encuentra concentrada en Brasil, Canadá y Estados Unidos. La región cuenta con 83.4 enfermeras por 10000 habitantes y México cuenta solamente con 23 enfermeras por 10000 habitantes.

Las enfermeras que fueron incorporadas al sistema de salud durante la contingencia epidemiológica lo hicieron a través de contratos temporales sin derechos laborales básicos, es decir, lo hicieron en condiciones de precariedad. La precariedad es una característica de la contratación de trabajadores de salud en México que alcanza a más de la mitad de las enfermeras del país3. Las condiciones de responsabilidad y de estrés durante este período laboral no han sido compensados por el tipo de contratación. Muy probablemente, la mayoría de estas enfermeras que terminarán sus contratos tendrán que buscar nuevas oportunidades si consideramos que el gobierno no tiene una política explícita de incremento de posiciones laborales en las instituciones públicas.

Es importante hacer notar que estos fenómenos no son exclusivos de México ya que se considera que a nivel mundial existe una escasez de trabajadores de la salud y, en algunos países en particular, de enfermeras. Sin embargo, a pesar de la reducción del gasto público en salud en los últimos diez años, México cuenta con los recursos públicos (290 USD al año per cápita 2019), para ser capaz de 
proteger a su fuerza laboral en salud, que como hemos señalado, representa el recurso más importante de cualquier sistema de salud4.

Las reformas del sistema de salud mexicano promovidas por su gran impulsor, Guillermo Soberón, son el espacio actual de práctica de las enfermeras mexicanas. Si bien la reforma en salud en México es un proceso inacabado, la enfermería se ha convertido en un agente clave en este proceso, y en los próximos años debe incrementar su capacidad para influir en las decisiones estratégicas, no sólo a partir de considerar que son el profesional de salud más abundante en el sistema, sino también porque con el tiempo han ganado espacios en posiciones de alta responsabilidad y desarrollado un liderazgo cada vez más conspicuo. Su formación, mezcla de conocimiento técnico/científico y humanista, las habilita para tomar decisiones basadas en una compresión holística de los individuos y las poblaciones que el sistema de salud atiende, planteamiento establecido y promovido por las organizaciones internacionales de salud. El futuro del sistema de salud es de la enfermería a nombre de la población que requiere atención.

\author{
G. Nigenda-López \\ ORCID: $0000-0003-0067-0664$ \\ Escuela Nacional de Enfermería y Obstetricia, \\ Universidad Nacional Autónoma de México, \\ Ciudad de México, México \\ gnigenda@comunidad.unam.mx
}

\title{
REFERENCIAS
}

1. Organización Panamericana de la Salud. Cerca de 570,000 trabajadores de la salud se han infectado y 2,500 han muerto por COVID-19 en las Américas. Washington, D.C.: OPS; 2020.

https://bit.ly/3covAtX

2. Organización Panamericana de la Salud. En medio de la pandemia de COVID-19, un nuevo informe de la OMS hace un llamamiento urgente a invertir en el personal de enfermería. Washington, D.C.: OPS; 2020. https://bit.ly/2Q_eUDAW

3. Aristizabal P, Nigenda G, Serván-Mori E. The precarization of the Mexican nursing labor market: a repeated cross-sectional analysis for the period 2005-2018. Hum Resour Health. 2019; 17(1): 1-9. https://doi.org/10.1186/s1296o-019-0417-x

4. Banco Mundial. Los países de Latinoamérica y el Caribe necesitan gastar más y mejor en salud para poder enfrentar una emergencia de salud pública como el COVID-19 de manera efectiva. Washington, D.C.: BM; 2020. https://bit.ly/315WKcB 